\title{
Aberrant Functional Connectivity in Dissociable Hippocampal Networks Is Associated with Deficits in Memory
}

\author{
Natalie L. Voets, ${ }^{1,2}$ Giovanna Zamboni, ${ }^{1}$ Mark G. Stokes, ${ }^{3}$ Katherine Carpenter, ${ }^{4}$ Richard Stacey, ${ }^{5}$ and Jane E. Adcock ${ }^{1,6}$ \\ ${ }^{1}$ FMRIB Centre, University of Oxford, John Radcliffe Hospital, Oxford OX3 9DU, United Kingdom, ${ }^{2}$ Oxford Epilepsy Research Group, Nuffield Department \\ of Clinical Neurosciences, John Radcliffe Hospital, Oxford OX3 9DU, United Kingdom, ${ }^{3}$ Oxford Centre for Human Brain Activity, University of Oxford, \\ Warneford Hospital, Oxford OX3 7JX, United Kingdom, and ${ }^{4}$ Russell Cairns Unit and Departments of ${ }^{5}$ Neurosurgery and ${ }^{6}$ Neurology, John Radcliffe \\ Hospital, Oxford OX3 9DU, United Kingdom
}

In the healthy human brain, evidence for dissociable memory networks along the anterior-posterior axis of the hippocampus suggests that this structure may not function as a unitary entity. Failure to consider these functional divisions may explain diverging results among studies of memory adaptation in disease. Using task-based and resting functional MRI, we show that chronic seizures disrupting the anterior medial temporal lobe (MTL) preserve anterior and posterior hippocampal-cortical dissociations, but alter signaling between these and other key brain regions. During performance of a memory encoding task, we found reduced neural activity in human patients with unilateral temporal lobe epilepsy relative to age-matched healthy controls, but no upregulation of fMRI signal in unaffected hippocampal subregions. Instead, patients showed aberrant resting fMRI connectivity within anterior and posterior hippocampal-cortical networks, which was associated with memory decline, distinguishing memory-intact from memory-impaired patients. Our results highlight a critical role for intact hippocampo-cortical functional communication in memory and provide evidence that chronic injuryinduced functional reorganization in the diseased MTL is behavioral inefficient.

Key words: fMRI; memory; plasticity; reorganization; temporal lobe epilepsy

\section{Introduction}

The potential to modulate human brain plasticity has received increasing interest as a means to promote functional recovery after injury (Stagg et al., 2012). The mechanisms underlying postinjury plasticity, and its impact on behavior, however, remain essentially unknown (Zatorre et al., 2012). Although the right hemisphere can adopt some language and motor functions following extensive damage early in brain development (VarghaKhadem et al., 1991; Holloway et al., 2000), anatomical and functional reorganization associated with chronic pain (Gustin et al., 2012; Makin et al., 2013) raises fundamental questions about the conditions promoting beneficial versus maladaptive plasticity.

In patients with unilateral medial temporal lobe (MTL) dysfunction, brain imaging studies reveal increased contralateral (Figueiredo et al., 2008; Banks et al., 2012), heightened ipsilateral (Powell et al., 2007; Schwarze et al., 2009), or solely decreased

Received Oct. 6, 2013; revised Feb. 1, 2014; accepted March 3, 2014.

Author contributions: N.L.V. and J.E.A. designed research; N.L.V., K.C., R.S., and J.E.A. performed research; N.L.V. analyzed data; N.L.V., G.Z., M.G.S., and J.E.A. wrote the paper.

This work was supported by the Medical Research Council (N.L.V.) and National Institute for Health Research Oxford Biomedical Research Centre (N.L.V. and G.Z.), and funding was provided by a Vera Down grant from the British Medical Association.

The authors declare no competing financial interests.

Correspondence should be addressed to Dr Natalie L. Voets, FMRIB Centre, University of Oxford, John Radcliffe Hospital, Headington, Oxford 0X3 9DU, UK. E-mail: natalie.voets@ndcn.ox.ac.uk.

DOI:10.1523/JNEUROSCI.4281-13.2014

Copyright $\odot 2014$ the authors $\quad 0270-6474 / 14 / 344920-09 \$ 15.00 / 0$
(Addis et al., 2007; Das et al., 2009; Voets et al., 2009; Bonelli et al., 2010; Bonnici et al., 2013) MTL neural activity during memory task performance. Furthermore, although increased activity has often been inferred to reflect compensatory functional reorganization, behavioral evidence has been mixed, demonstrating both a positive (Figueiredo et al., 2008) and negative (Powell et al., 2007) impact of upregulated contralateral MTL neural activity on memory function. Critically, episodic memory declines following unilateral MTL surgery are most reliably predicted by the magnitude of preoperative activity in to-be-removed, rather than contralesional MTL structures (Rabin et al., 2004; Powell et al., 2008; Bonelli et al., 2010), challenging the concept of effective memory reorganization.

To date, studies of memory (re)organization have assessed the hippocampus as a unitary structure. Yet, anatomical connectivity, gene expression profiles and electrode recordings indicate distinct contributions of hippocampal subregions to memory formation and retrieval in both rats and nonhuman primates (Moser and Moser, 1998; Fanselow and Dong, 2010). Brain imaging techniques identify similar dichotomies within human MTL structures, implying the existence of potentially distinct hippocampal memory systems (Aggleton, 2012). In particular, the anterior and posterior hippocampus have selective connections with separate polymodal cortical networks (Kahn et al., 2008; Poppenk and Moscovitch, 2011; Libby et al., 2012) indicating distinct routes of information flow likely supporting qualita- 
Table 1. Demographic and clinical data

\begin{tabular}{|c|c|c|c|c|c|c|}
\hline Group & Men & Age & $\begin{array}{l}\text { Seizure } \\
\text { onset }\end{array}$ & $\begin{array}{l}\text { Epilepsy } \\
\text { duration }\end{array}$ & $\begin{array}{l}\text { Febrile } \\
\text { convulsion }\end{array}$ & $\begin{array}{l}\text { Hippocampal } \\
\text { sclerosis (MRI) }\end{array}$ \\
\hline Controls $(n=25)$ & 15 & $31.7 \pm 7(19-49)$ & - & - & - & - \\
\hline $\operatorname{LTLE}(n=17)$ & 8 & $32.2 \pm 9.4(18-49)$ & $14.6 \pm 9.7(1-36)$ & $17.4 \pm 12.3(5-46)$ & $8 / 17$ & $6 / 17$ \\
\hline $\operatorname{RTLE}(n=14)$ & 8 & $36.6 \pm 9.1(23-51)$ & $16.6 \pm 10.1(3-35)$ & $21.4 \pm 8(8-33)$ & $5 / 14$ & $5 / 14$ \\
\hline
\end{tabular}

Age, age at seizure onset, and duration of epilepsy are presented in mean \pm SD (range) years.

tively different memory computations (Vincent et al., 2006; Poppenk et al., 2010; Gour et al., 2011; Hirshhorn et al., 2012). This raises the intriguing possibility that postinjury plasticity could occur selectively within functionally dissociable hippocampal regions, potentially contributing to diverging findings in previous studies.

Here, we tested this possibility by investigating the organization of dissociated hippocampal memory circuits and their relation to memory performance using task-based and resting functional MRI measures in healthy volunteers and patients with unilateral temporal lobe epilepsy. Recordings from surgically implanted electrodes (Kahane and Bartolomei, 2010; Gour et al., 2011) and the success of anterior MTL neurosurgery for controlling seizures (Clusmann et al., 2002) support a preferential involvement of anterior MTL structures in seizure activity. These patients, therefore, offer an ideal model in which to test the hypothesis that postinjury plasticity occurs selectively within anterior and posterior hippocampal "networks," uniquely contributing to adaptive reorganization or memory decline.

\section{Materials and Methods}

Participants. Thirty-one patients (16 men) with unilateral temporal lobe epilepsy (TLE) were recruited through the Oxford Epilepsy Surgery Program. Patients were aged 18-51 (mean, 34.2) and had seizures arising unilaterally from the left $(n=17)$ or right $(n=14)$ MTL based on comprehensive clinical assessment including video-telemetry, highresolution MRI, neuropsychological evaluation, and where necessary, implanted electrode recordings. Patients were right-handed except for one left-TLE and one right-TLE patient who were both left-handed. Clinical MRI was normal or equivocal in 11 patients (6 left -TLE) and revealed reduced hippocampal volumes consistent with hippocampal sclerosis $(n=18,10$ left-TLE) or anterior MTL focal cortical dysplasia ( $n=2,1$ left-TLE). One right-TLE patient had a small cavernoma in the anterior temporal lobe in addition to hippocampal sclerosis. History of an initial precipitating injury (febrile convulsion) was documented in $n=13 / 31$ patients (Table 1 ). All patients were taking varied combinations of antiepileptic medication (on average 3 of 14 possible drugs). Twenty-five right-handed healthy volunteers ( 15 men) with no neurological or psychiatric history, age-matched to the patients (range, 19-49; mean, 31.7 ; independent samples $t$ test $p=0.3$ ) were also studied. Informed written consent was obtained from all participants. The study was approved by the South London Research Ethics committee.

Complex scene encoding task. Before the scan, participants were familiarized with a set of eight complex color scenes depicting landscapes, building, or animals (Voets et al., 2009) a total of seven times in random order. Images were presented for $4 \mathrm{~s}, 1 \mathrm{~s}$ apart. Subsequently, during the fMRI scan, participants viewed alternating blocks of novel scenes or randomly ordered "familiar" scenes across 10 blocks, separated by 15 s fixation periods. Participants were asked to indicate using a button-box whether each scene contained an animal (to facilitate encoding) and were told there would be a recognition test after the scan. Using this robust block-design, we were able to identify regions showing an increased neural response during encoding of new scenes into memory compared with viewing of familiar scenes. Task-fMRI data were excluded for nine patients due to absence of activation surviving statistical threshold within the MTL for the encoding contrast (new > old scenes). Statistically significant activation for main effects of viewing scenes was present in all but four patients. Behavioral recognition data, acquired $\sim 30$ min after the encoding task and outside of the scanner, were available for only 15/25 controls and 21/31 patients. Analyses relating fMRI data with performance scores, therefore, instead used clinical neuropsychological test scores.

Magnetic resonance imaging. Magnetic resonance images were acquired on a 3T Siemens Verio scanner using a 32-channel head coil. Resting T2*-weighted Blood Oxygen Level Dependent (BOLD) data were acquired using an echo-planar imaging sequence $(\mathrm{TR}=3.5 \mathrm{~s}, \mathrm{TE}=$ $30 \mathrm{~ms}$, slice thickness $=2 \mathrm{~mm}$, 54 slices, voxel size $2 \times 2 \times 2 \mathrm{~mm}, 5 \mathrm{~min}$ acquisition) in 29/31 patients. Participants were instructed to lie still with their eyes closed while remaining awake. T1-weighted images were acquired using a 3D MPRAGE sequence, providing isotropic voxels of $1 \times$ $1 \times 1 \mathrm{~mm}^{3}$. For the encoding task (always acquired after the resting scan), BOLD-fMRI T2*-weighted echo-planar images were acquired continuously during performance of the complex scene-encoding task in the scanner. The fMRI parameters were as follows: $\mathrm{TR}=3 \mathrm{~s}, \mathrm{TE}=28 \mathrm{~ms}$, 44 slices, $3 \mathrm{~mm}$ slice thickness, voxel size: $3 \times 3 \times 3 \mathrm{~mm}, 181$ volumes, 9 min acquisition. Three "dummy" volumes at the start of the fMRI scan were rejected to allow magnetization equilibrium.

Memory task analysis. Data were preprocessed and analyzed using tools from the FMRIB Software Library. Preprocessing included correction for head motion, correction for geometrical distortions at air tissue boundaries using fieldmaps, spatial smoothing using a $5 \mathrm{~mm}$ full-width halfmaximum Gaussian kernel, and high-pass filtering (100 s) to reduce low-frequency artifacts. Regions showing increased BOLD signal when participants viewed novel scenes compared with familiar scenes (i.e., encoding-related processing) were identified at the individual-subject level using general linear models implemented in FMRIB's Expert Analysis Tool (FEAT). Activation maps were generated using cluster statistics performed on all voxels above a threshold of $z=2.3$ with a corrected cluster extent of $p<0.05$. Physiological fluctuations due to respiratory and cardiac activity were recorded using respiratory bellows and a pulse oximeter during the scan and modeled as confound regressors (Brooks et al., 2008). Whole-brain activation maps were first generated to visually inspect for head motion-related artifacts and regress out motion-related time-series or head-motion outlier volumes as necessary. However, as we wished to test for adaptive activation-related changes within MTL structures, and whole-brain multiple-comparison corrections are overly stringent for this question, we report results from additional analyses restricted to a bilateral atlas-based MTL mask applied to subject-specific and group-level analyses. The MTL region of interest was defined on the MNI152 brain template combining the hippocampus (HPC) and posterior parahippocampal gyrus (PHG) probabilistic labels from the Harvard-Oxford atlas with the entorhinal cortex (EC) label (incorporating the perirhinal cortex) from the Juelich histological atlas. The hippocampal template mask was thresholded at $50 \%$ and the cortical labels at $35 \%$ to restrict these to gray matter. The resulting bilateral ROI was applied to each subject's fMRI data to constrain cluster-wise statistics. Group-level general linear models were used to identify voxels showing increased neural signal during scene encoding (new $>$ old scenes) in left-sided or right-sided patients compared with controls.

Magnitudes of fMRI activation (mean BOLD percentage signal change) during encoding were extracted using Featquery at the singlesubject level from the left and right hemisphere parahippocampal and entorhinal template regions separately as well as from the anterior and posterior hippocampal functional divisions identified from resting fMRI analyses as detailed below.

Resting analysis: functional divisions. Previous studies have used anatomical boundaries to divide the hippocampus into anterior and poste- 
rior segments. It is not evident that such anatomical borders will correspond well to functional divisions, especially in chronic patient populations. Therefore, we adopted a novel approach to divide the hippocampus according to preferred functional connections (from baseline resting fMRI) with cortical regions (Zarei et al., 2012). We wished to identify functional divisions within the hippocampus unbiased by subjective anatomical delineations or group status (patient vs control) for subsequent analysis of memory task-related activation and performance.

Resting fMRI data were preprocessed in the same way as task-fMRI data. A seed-based correlation approach (SBCA) was used to correlate resting fMRI signals between anatomically defined regions (O'Reilly et al., 2010) to functionally isolate hippocampal subregions. For this analysis, the resting fMRI signal from every voxel in the template hippocampal atlas mask was correlated with the characteristic resting fMRI signal from two hemisphere-lateralised sets of cortical regions previously reported to form anterior and posterior "memory networks" (Kahn et al., 2008; Poppenk and Moscovitch, 2011; Libby et al., 2012). The time courses representing head motion, CSF and white matter were regressed out during analyses to reduce the influence of nontissue related confound effects. The cortical network regions were selected from the Harvard-Oxford and Juelich probabilistic standard-brain atlases. According to the functional network connections reported in the publications above, we selected the entorhinal cortex (including the perirhinal cortex), orbitofrontal cortex, and temporal pole to form the anterior "memory network" regions and the parahippocampal gyrus, posterior cingulate/ precuneus, thalamus, dorsolateral prefrontal cortex, and combined lingual/fusiform regions to form "posterior memory network" regions (Fig. 1b). The dorsolateral prefrontal cortex was formed by combining superior and middle frontal gyrus atlas regions corresponding grossly to areas 9 and 9/46 as described by (Petrides and Pandya, 1999). All atlasbased masks were nonlinearly registered to each subject's highresolution structural image and masked to exclude voxels falling in CSF based on automated individual subject tissue segmentations generated using FMRIB's Automated Segmentation Tool. The hippocampal "seed" mask and the cortical "target" masks were finally linearly aligned to the resting fMRI data for analysis using a boundary-based optimized registration method (Greve and Fischl, 2009) implemented in FEAT.

For every subject, SBCA produced a spatial correlation map in which each voxel in the whole hippocampal atlas mask was labeled according to preferential signal correlations with each cortical network region. To verify whether in patients, the anterior-posterior functional division within the hippocampus was preserved, we visually inspected average correlation population maps generated for an age-matched subgroup of 25 of our TLE patients and our 25 controls separately. For this purpose, we summed the correlation maps for controls and patients and then thresholded these to retain only those voxels consistently labeled as posterior or anterior in half of that population (12/25 controls and 12/25 patients). Following confirmation that functional divisions globally matched between groups, we combined all correlation maps to identify the $z$-and $y$-axis coordinates along which to divide the atlas hippocampal mask into anterior (aHPC) and posterior (pHPC) segments for subsequent analyses (Fig. 1a, bottom row).

Resting analysis: correlation magnitudes. In the first resting fMRI analysis detailed above, we identified where the functional boundary within the hippocampus occurred. In a second resting fMRI analysis, we quantified the strength of functional signal correlations from the functionally defined anterior and posterior hippocampal segments, separately, with each of its corresponding cortical targets in all available patients $(n=29)$ and controls $(n=25)$. The goal of this analysis was to test for differences between patients and controls in the strength of interregional functional connections, identify which specific regions are affected, and whether the strength of baseline functional connections relates to task activity and performance. SBCA was therefore repeated for every participant using the anterior and posterior hippocampal segments in each hemisphere, derived from the first analysis, as two separate "seeds" and each of the cortical regions as targets. For this analysis, the pooled group average anterior and posterior segmentations were first nonlinearly aligned to each individual's anatomical brain imagine, and CSF voxels were removed to constrain sampling to the gray matter volume for that subject. The resulting individual-specific masks were then aligned to the functional data to conduct functional connectivity analyses. The result of the second stage SBCA analysis was a single value for every voxel in the anterior or posterior hippocampal mask representing its partial correlation with each cortical target in the corresponding hemisphere (accounting for the magnitude of correlation with every other cortical target). From this, we calculated a single mean correlation score ( -1 to 1$)$ for every subject between each hemisphere-lateralized cortical mask and the anterior and posterior hippocampal segment, separately (producing 16 hemisphere-specific mean correlation values per subject; i.e., 8 cortical masks tested twice, once with the anterior and once with the posterior hippocampal segment).

Neuropsychology. Every patient underwent a comprehensive neuropsychological assessment including: tests of general intellectual ability and attentional/executive function from the Wechsler Adult Intelligence Scale (WAIS-IV), tests of verbal and nonverbal memory abilities using story and figure immediate and delayed recall as well as list and design learning and recall from the Brain Injury Rehabilitation Trust Memory and Information Processing Battery. We selected story and figure immediate recall as measures of verbal and nonverbal retention abilities on 
Table 2. Hippocampal-cortical resting correlation values

\begin{tabular}{|c|c|c|c|c|c|c|c|c|}
\hline & \multicolumn{3}{|l|}{ Anterior } & \multicolumn{5}{|l|}{ Posterior } \\
\hline & $\mathrm{EC}$ & TP & OFC & PHG & T0 & $\mathrm{PCC}$ & DLPFC & THAL \\
\hline \multicolumn{9}{|c|}{ Left hemisphere } \\
\hline Controls & $0.16 \pm 0.11$ & $0.05 \pm 0.09$ & $0.05 \pm 0.07$ & $0.09 \pm 0.05$ & $0.01 \pm 0.04$ & $0.02 \pm 0.05$ & $-0.03 \pm 0.04$ & $0.04 \pm 0.04$ \\
\hline LTLE & $0.24 \pm 0.14$ & $0.08 \pm 0.09$ & $0.01 \pm 0.07$ & $0.14 \pm 0.08$ & $0.05 \pm 0.05$ & $-0.03 \pm 0.04$ & $0.00 \pm 0.05$ & $0.02 \pm 0.07$ \\
\hline RTLE & $0.14 \pm 0.13$ & $0.08 \pm 0.12$ & $0.02 \pm 0.08$ & $0.11 \pm 0.06$ & $0.03 \pm 0.06$ & $0.02 \pm 0.07$ & $-0.03 \pm 0.03$ & $0.03 \pm 0.05$ \\
\hline \multicolumn{9}{|c|}{ Right hemisphere } \\
\hline Controls & $0.20 \pm 0.12$ & $0.02 \pm 0.1$ & $0.03 \pm 0.07$ & $0.06 \pm 0.05$ & $0.01 \pm 0.05$ & $0.03 \pm 0.06$ & $-0.02 \pm 0.06$ & $0.03 \pm 0.05$ \\
\hline LTLE & $0.22 \pm 0.15$ & $0.04 \pm 0.11$ & $0.03 \pm 0.1$ & $0.12 \pm 0.1$ & $0.03 \pm 0.05$ & $0.01 \pm 0.06$ & $-0.04 \pm 0.04$ & $0.05 \pm 0.04$ \\
\hline RTLE & $0.18 \pm 0.14$ & $0.05 \pm 0.15$ & $0.01 \pm 0.11$ & $0.11 \pm 0.07$ & $0.03 \pm 0.08$ & $-0.01 \pm 0.06$ & $-0.02 \pm 0.05$ & $0.04 \pm 0.05$ \\
\hline
\end{tabular}

Mean resting correlation values \pm SD between hemisphere-specific functionally-defined anterior and posterior hippocampal masks (Fig. 1a) and every associated cortical "memory network" region from 25 healthy controls and 29 patients with unilateral temporal lobe epilepsy (17 left-TLE and 12 right-TLE).

which to separate memory-intact (clinical range Z-scores down to -1.33 ) from memory-impaired patients ( $Z$-scores of -1.34 and below).

Statistical analyses. Nonimaging-based statistical analyses were conducted using SPSS v21. Multivariate ANOVAs were used to test for between-group differences on imaging-based measures comparing: volumes of each of the left and right hemisphere hippocampal functional subregions; mean resting fMRI signal correlation between anterior hippocampal segments and associated anterior cortical regions as well as between posterior hippocampal segments and associated posterior cortical regions; and mean BOLD fMRI percentage signal change for the contrast "new scenes > old scenes" (reflecting encoding processes) from each of the left and right hemisphere MTL regions. Each of these imaging measures was also compared in MANOVAs between patients deemed "borderline" or impaired on clinical neuropsychology measurements. Paired-samples $t$ tests were used to compare hippocampal-cortical resting correlation values between the left and right hemispheres within controls. Relationships between behavioral and imaging measures were established using two-tailed Pearson's correlations.

\section{Results}

Preservation of hippocampal functional boundaries in chronic seizure patients

To determine the impact of anterior MTL seizures on hippocampal functional distributions, we first identified hippocampal boundaries functionally in balanced groups of 25 healthy controls and 25 age-matched patients with unilateral temporal lobe epilepsy using resting functional MRI. Each voxel in the hippocampus was labeled automatically according to whether it had preferential resting fMRI signal correlations with anterior or posterior cortical regions previously reported to form dissociable memory networks (Kahn et al., 2008; Poppenk and Moscovitch, 2011; Libby et al., 2012). Population average maps revealed a boundary dividing the hippocampus along the longitudinal axis into a large posterior segment and a smaller anterior border in both patients and controls (Fig. 1a, top and middle rows). The location of the functional border along the anterior-posterior $(y)$ and inferior-superior $(z)$ axes globally matched between patients and controls; i.e., functional segregations along the anterior-posterior axis of the hippocampus are preserved despite chronic MTL injury in TLE. To quantify regional brain activity during a subsequent memory task, we therefore used the average $y$ - and $z$-axis coordinates to define a functional hippocampal border common to patients and controls (Fig. 1a, bottom row).

In patients, volumes of the functionally defined posterior hippocampus on the epileptic side were reduced compared with controls (left TLE patients: $F=19.35, p<0.001$; right TLE patients: $F=31.22, p<0.001)$. There was a trend for anterior hippocampal volumes ipsilateral to the side of seizures to be reduced as well in left TLE patients $(F=3.48, p=0.069)$. For all subsequent analyses, these functionally defined hippocampal masks were therefore registered to each subject's structural image and constrained to gray matter before extracting resting connectivity or neural activity values.

\section{Aberrant functional connectivity in dissociated hippocampal networks in chronic epilepsy}

We next tested whether the strength of neural signaling differed among regions forming anterior and posterior memory networks between patients and controls. In healthy controls $(n=25)$, hippocampal-cortical mean resting signal correlations did not differ between the left and right hemispheres (all paired-sample $t$ test values $>0.1$ ). To quantify resting connectivity abnormalities common across TLE patients, we therefore averaged the values from both hemispheres in controls for comparison against correlation values from the affected (whether left or right) and unaffected hemispheres of patients. Relative to controls, patients $(n=29)$ showed reduced spontaneous signal correlations between the affected posterior hippocampus and posteromedial cortex (posterior cingulate/precuneus; $F=12.19, p=0.001$; Table 2). Conversely, patients showed abnormally increased functional correlations between the posterior hippocampus and parahippocampal gyrus both ipsilaterally $(F=9.06, p=0.004)$ and contralateral to the side of seizure focus $(F=4.24, p=0.045$; uncorrected for multiple comparisons). In subsequent groupspecific post hoc analyses, comparing left-TLE and right-TLE patients separately against controls, hippocampal-cortical resting correlation values were assessed separately for each hemisphere. Group-specific analyses revealed the above findings to be primarily driven by the larger group of left-TLE patients $(n=17)$. In the left-TLE group, in addition to reduced left posterior hippocampalposteromedial $(F=11.29, p=0.002)$ and bilaterally increased posterior hippocampal-parahippocampal signal correlations (left: $F=7.36, p=0.01$; right: $F=4.9, p=0.032$ ), we also observed heightened functional connectivity in the affected hemisphere between the anterior hippocampus and entorhinal cortex $(F=5.72, p=0.022)$. Signal from each cortical region was entered into partial correlations twice (once with the anterior and once with the posterior hippocampal region of the corresponding hemisphere; Fig. 2a). Left-TLE group differences in ipsilateral posterior hippocampal-posteromedial and posterior hippocampalparahippocampal connectivity as well as heightened anterior hippocampal-entorhinal connectivity (Fig. $2 b, c$ ) survive Bonferroni correction for two comparisons.

\section{Interplay between hippocampal-cortical functional integration and memory task activity}

To test whether heightened resting connectivity between posterior hippocampal-cortical regions is functionally reflected in hyper-activity during memory encoding, we compared neural activity in MTL regions during performance of a complex scene memory-encoding task between patients and controls. To avoid 
misinterpreting an absence of reorganization in patients not fully engaged in the task or unable to perform it, we restricted this analysis to participants showing neural activity during encoding (fMRI signal to new images $>$ old images) surviving a cluster-wise correction to $p<0.05$ within the MTL region of interest at the individual subject level ( $n=22$ patients, all controls).

As expected, during performance of the complex scene encoding fMRI task, healthy volunteers activated all MTL structures bilaterally, showing the greatest signal increase during encoding in the posterior parahippocampal gyrus (Fig. $3 a$ ). The average group map for right-TLE patients revealed activation only in the left MTL, whereas the left-TLE average group map showed activation of the right MTL and posterior left MTL (Fig. 3b,c).

To quantify whether fMRI signal magnitudes during scene encoding might be redistributed across MTL subregions in patients, we extracted the mean BOLD signal from the functionally defined anterior and posterior hippocampal segments as well as atlas-based entorhinal and posterior parahippocampal regions-of-interest in both hemispheres. Although the magnitude of neural activity did not differ between patients and controls in the anterior or posterior hippocampal regions during scene encoding, patients showed reduced BOLD signal in the PHG both on the affected side (left-TLE: $F=8.36, p=0.006$; right-TLE: $F=$ 8.15, $p=0.008$ ) and contralaterally (left-TLE: $F=8.72, p=$ 0.006 ; right-TLE: $F=6.95, p=0.013$; Fig. $3 d$ ). We found no evidence or indication for upregulated activity, hypothesized to sustain memory encoding in our patients, within either the affected posterior hippocampus or unaffected (contralateral) MTL structures relative to healthy controls.

All patients during the study were taking between two and four antiepileptic drugs (in variable combinations/doses among 14 different prescribed medications). Certain antiepileptic drugs are reported to alter the $\mathrm{fMRI}$ BOLD signal. In particular, carbamazepine may diminish fMRI activation during memory processing (Jokeit et al., 2001). To explore whether this drug's adverse effects on fMRI signal could mask subregional increases in hippocampal activity during scene encoding, we repeated between-group fMRI signal magnitude comparisons after excluding patients prescribed carbamazepine $(n=4$ right-TLE, $n=$ 8 left-TLE). Noting the reduced patient sample sizes, this analysis replicated the larger group findings of bilaterally reduced parahippocampal BOLD signal, but again did not provide any indication for increased activity with MTL subregions in TLE patients.

It is possible that compensatory upregulation of MTL structures could occur in early phases following the onset of seizures and decay as seizure activity becomes chronic over many years. In this case, neural activity during memory encoding would be expected to decline with increasing disease duration. We did find an association between disease duration and the magnitude of the fMRI signal in the posterior affected hippocampus. However, neural activity during encoding in this region increased rather than decreased with disease duration $(r=0.48, p=0.024)$, and was no longer significant when accounting for age variability within the patient group (all partial correlation $p$ values $>0.2$ ). Thus, patients with shorter disease durations did not recruit unaffected MTL structures to a greater extent than patients with long-established epilepsy.

Behavioral correlates of hippocampal network (dys)function Although we did not detect increased neural activity on our scene encoding task, it remains possible that altered resting functional connectivity within the MTL may reflect functional reorganization not captured through the fMRI task (for example because encoding was already deficient in our patients).

If altered resting functional connectivity reflects compensatory efforts to preserve functions at risk from epileptic disruption, then these changes should be observed in patients with relatively intact memory performance and not in patients who are already memory-impaired. To test this, we classified patients according to whether or not they showed impairment on relevant clinical measures of memory performance.

Recall of verbal and visuospatial information is commonly impaired by unilateral temporal lobe surgery (for review, see Helmstaedter, 2004). We therefore divided patients into two groups according to whether their neuropsychological test performance on figure immediate recall (in right-TLE patients) or story immediate recall (in left-TLE patients) was intact (normalized $\mathrm{Z}$-scores ranging from positive down to -1.33 , clinical borderline cutoff range) or impaired (normalized $z$-score of -1.34 or below). We then determined whether MTL volumes, taskfMRI encoding-related activity, or resting baseline functional connectivity differentiated recall-impaired from recall-intact patients. Recall-impaired and memory-intact patients did not differ 

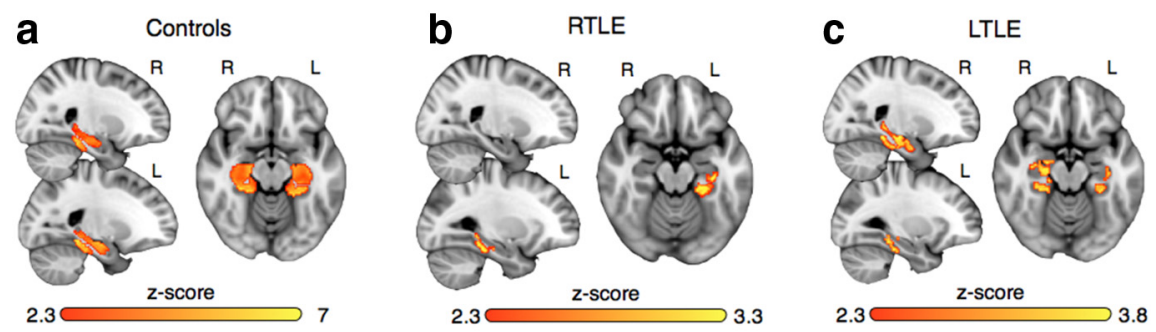

d Mean fMRI signal during scene encoding
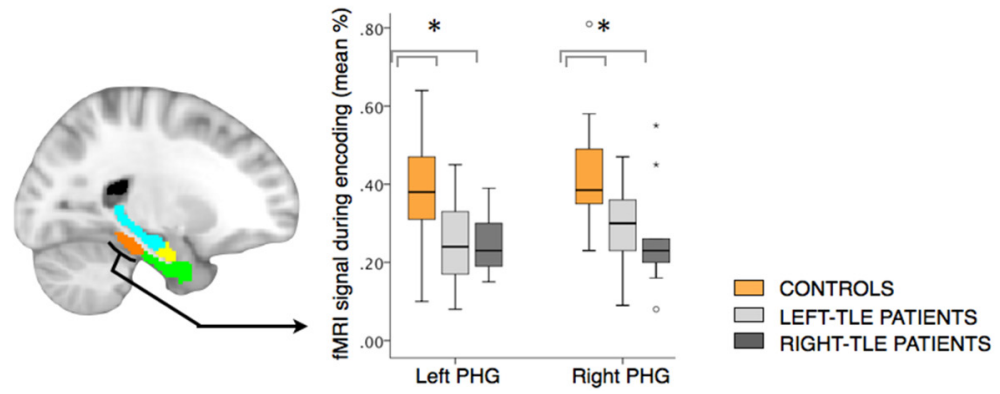

Figure 3. Medial temporal lobe fMRl activation during complex scene encoding. Region-of-interest constrained general linear model analysis of increased brain activity during encoding of novel relative to viewing familiar scenes in healthy controls $(\boldsymbol{a})$ and patients with chronic unilateral medial temporal lobe epilepsy $(\boldsymbol{b}, \boldsymbol{c}$; maps depicting activation thresholded at $z$-score of 2.3 , cluster-wise corrected to $p<0.05$ ). Comparison of mean bold signal change during encoding sampled from each of the MTL subregions revealed significantly reduced neural activity in the parahippocampal gyrus (d) in patients compared with controls bilaterally). Asterisks denote significant between-group differences in mean BOLD fMRI signal $(p<0.05)$. We found no evidence for "compensatory" increased activation during memory encoding across either ipsilateral or contralateral memory systems in chronic epilepsy. TLE, Temporal lobe epilepsy; PHG, parahippocampal gyrus.

on MTL volumetric measures or neural activation during memory encoding. However, surprisingly, memory-impaired patients had significantly higher ipsilateral anterior hippocampal-entorhinal resting functional connectivity $(F=4.44, p=0.045$; Fig. $4 a)$ and lower contralateral hippocampus-posterior cingulate $(F=8.36$, $p=0.008)$ resting signal correlations relative to memory-intact patients (Fig. 4b). The observed bilateral increases in posterior hippocampal-parahippocampal resting connectivity did not distinguish patients with and without clinical memory impairment due to one left-TLE outlier with high contralateral resting connectivity but intact story recall. Removing this patient, heightened contralateral (but not ipsilateral) posterior hippocampalparahippocampal resting connectivity was also higher in memory-impaired than in memory-intact patients $(F=6.88, p=$ $0.015)$, with entorhinal and posterior-cingulate findings unaffected/strengthened ( $p=0.008$ and $p=0.009$, respectively). Thus, aberrantly increased hippocampal-cortical functional connectivity in the seizure-generating MTL memory network is not efficiently compensatory, but instead reflects disrupted memory performance.

\section{Discussion}

Neural plasticity underlies fundamental brain processes ranging from normal learning to adaptive changes following injury. Nonetheless, it remains unknown whether, and under which circumstances, microstructural and functional remappings following neuronal damage are compensatory, adaptive, incidental, or maladaptive (Saur and Hartwigsen, 2012). Recent evidence of functional dissociations among hippocampal memory systems (Aggleton, 2012) provides a novel framework to determine MTL compensatory capacities central to understanding mechanisms affecting memory decline in health and disease.
Our aim was to test the impact of chronic unilateral seizures on the distribution and function of anatomically segregated hippocampal memory networks. Contrary to what might have been expected, we demonstrate remarkably preserved hippocampal functional dichotomies, matching known anatomical connection patterns (Moser and Moser, 1998), in patients with long-standing seizures (mean, 19 years). Despite preserved functional boundaries, however, we found no evidence that patients upregulate neural activity either between the affected and unaffected MTLs or between anterior and posterior hippocampal systems during memory encoding. Highresolution MTL imaging data report similar findings from subregions of the epileptic and contralateral hippocampus (Das et al., 2011) that are difficult to reconcile with theories of adaptive compensation (Alessio et al., 2013). Instead, spontaneous hippocampal-cortical functional connectivity in patients with chronic seizures was dramatically different from control subjects within the dissociable memory systems. The degree of both pathological hyperconnectivity and hypoconnectivity reflected memory impairment, rather than preservation of function: the strongest functional signaling abnormalities were seen in patients with the greatest memory impairments. Our findings raise the possibility that, in the presence of active disease processes, macroscopic fMRI markers of MTL plasticity reflect pathological influences on neuronal signaling rather than efficient compensatory remappings supporting preserved memory functions.

In both patients and controls, within the MTL, anterior hippocampal resting signals correlated most strongly with those in entorhinal cortex, whereas the posterior hippocampus showed preferential connectivity with the parahippocampal gyrus (Table 2). These circuits correspond well to anatomical connections (Blatt and Rosene, 1998) reported in nonhuman animals. Additionally, patterns of extra-MTL functional connections replicated previously reported anterior-posterior gradients in healthy volunteers (Kahn et al., 2008; Poppenk and Moscovitch, 2011; Libby et al., 2012) matching preferential anatomical projections from the anterior hippocampus to the temporal pole, amygdala, and prefrontal cortex, and from the posterior hippocampus to posterior cingulate and visual association areas (for review, see Aggleton, 2012). Human functional imaging studies implicate these same cortical regions during distinct phases of episodic memory processing, forming putative anterior and posterior memory networks (Vincent et al., 2006; Huijbers et al., 2011; Sestieri et al., 2011).

Within the dissociated hippocampal-cortical networks, resting connectivity was altered primarily within the epileptic MTL. Our data corroborate previous evidence for a selective vulnerability of functional connections between the hippocampus and posterior cingulate/precuneus regions of the "default mode" network (DMN) in epilepsy. The strength of hippocampal- posterior DMN functional connectivity reflects memory performance in both healthy aging (Wang et al., 2010) and disease (Vann et al., 
a

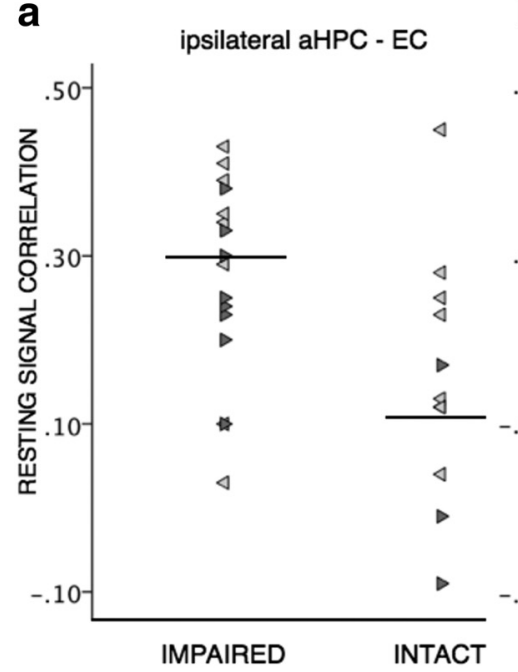

b

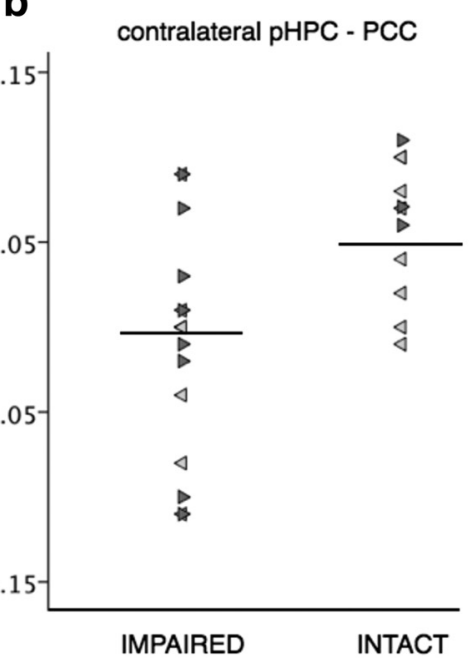

C

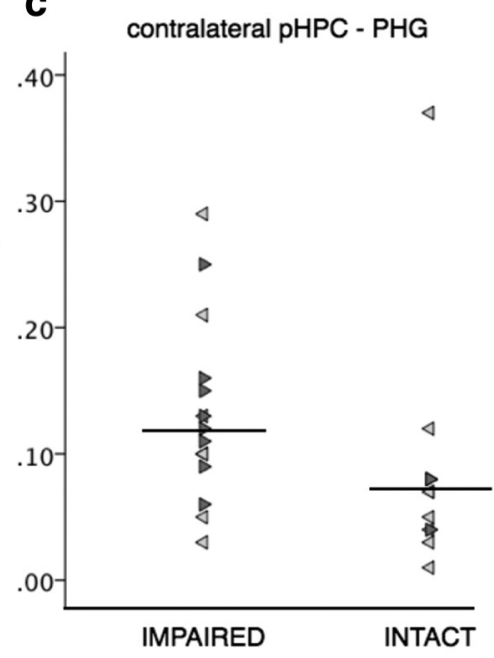

Figure 4. Neural correlates of memory impairment. Direct comparison of patients intact or impaired on clinical neuropsychological tests of recall revealed that patients with the most deviant hippocampal-cortical functional connectivity suffered severely impaired memory. Both abnormally heightened neural fMRI signal correlation between the epileptic aHPC and the EC ( $\boldsymbol{a}$ ) as well as reduced functional connectivity between the contralateral $\mathrm{PHPC}$ and $\mathrm{PCC}(\boldsymbol{b})$ were significantly more pronounced in memory-impaired patients than in patients with intact memory performance ( $p=0.029$ and $p=0.005$, respectively). Abnormally high resting connectivity between the contralateral pHPC and PHG (c) also differentiated between impairment groups when removing the single left TLE outlier ( $p=0.006$ removing the outlier). These results demonstrate altered hippocampal-cortical neural signaling in response to chronic disease does not support efficient reallocation of memory function but more likely reflect nonspecific reactions to pathological processes in chronic disease. PHG; Parahippocampal gyrus; EC, entorhinal cortex; PCC, posterior cingulate and precuneus cortex. Light gray arrows represent left-TLE patients; dark gray arrows represent right-TLE patients.

2009; Alessio et al., 2013). In the first study to directly relate MTL-DMN functional connectivity with memory performance in epileptic patients, reduced signal correlations between these regions on the epileptic side reflected reduced performance on material-specific recognition tests (McCormick et al., 2013). In our patients, contralateral posterior hippocampal-DMN resting connectivity was lowest in patients who were impaired on memory testing, presumably reflecting a cumulative effect on top of significant ipsilateral hypoconnectivity across the TLE group. As hippocampal-DMN functional connections are affected by subclinical epileptic discharges (Laufs et al., 2007), it is difficult to differentiate the effect on memory function of epilepsy-related fiber atrophy (Concha et al., 2005) from interictal discharge spread (Laufs et al., 2007). However, aberrant hippocampalDMN functional connectivity clearly did not benefit but was instead associated with impaired memory function in our study, consistent with the findings of McCormick et al. (2013).

Our results extend this prior study by determining the behavioral correlates of plasticity across the entire, functionally distinct anterior and posterior hippocampal-cortical networks. Altered anterior hippocampal-entorhinal functional coupling has previously been noted in TLE, albeit anti-correlated (Bettus et al., 2010) with electrophysiological indications of increased connectivity within the epileptic zone (Bettus et al., 2011). The reason for discrepancies in the reported directionality of implanted EEG signaling (Bettus et al., 2011; He et al., 2008), and thus, the correspondence between $\mathrm{PMRI}$ and EEG findings, requires further investigation. However, methodological differences in the computation of signal correlations and data sampling rates, and nonsimultaneous data acquisition complicate direct comparison of implanted EEG and fMRI signals (Bettus et al., 2011). Critically, we demonstrate that increased anterior hippocampalentorhinal functional connectivity reflects impairment rather than preservation of memory functions. A similar trend was seen in the posterior MTL network, in which aberrantly high posterior hippocampal-parahippocampal resting connectivity contralat- eral to the seizure focus was associated with impaired memory performance. These findings demonstrate that, in addition to functional decoupling between brain regions, pathological hyperconnectivity may equally reflect behavioral impairments in chronic disease.

Mirroring recent evidence in Alzheimer's disease (AD), our results suggest that macrostructural measures of neural plasticity in chronic epilepsy may reflect an unspecified response to pathology and are not functionally efficient. Imaging studies have revealed increased neural activity in key MTL memory structures in patients at risk of $\mathrm{AD}$, but extensively deficient MTL activity in patients with probable AD (for review, see Ewers et al., 2011), even when controlling for MTL atrophy (Zamboni et al., 2013). It has been advanced that hyperactivation in presymptomatic $\mathrm{AD}$ phases may reflect compensatory efforts that concede to hypoactivation as neurodegeneration progresses in the MTL (Putcha et al., 2011). However, excessive MTL neural activity appears nonspecific for successful memory performance (Zamboni et al., 2013), although pharmacological normalization of hippocampal hyper-activation can improve memory function (Bakker et al., 2012). These data are difficult to reconcile with a proposed behaviorally beneficial role for early phase heightened neural response. The strength of our data lies in the ability to probe measures of plasticity in MTL networks directly, specifically, and unilaterally affected by seizure activity. Multiple pathological processes are known to co-occur in TLE, including subclinical abnormal electrical discharges (Sperling and O'Connor, 1990), hippocampal and neocortical sclerosis (Thom et al., 2009), dentate gyrus axonal ("mossy fiber") sprouting (Sutula et al., 1989; Proper et al., 2000), degeneration of fiber bundles associated with seizure spread (Concha et al., 2005; Focke et al., 2008), and neurodegeneration of their cortical targets (Bernhardt et al., 2009). In addition, neurodevelopmental features have been noted during histological analysis of surgical tissue samples (Johns and Thom, 2008) and on recent brain imaging data (Voets et al., 
2011). The specific effect of these individual processes on functional MRI signals is currently poorly understood.

Our results do not rule out adaptive changes across widespread functional networks involving the hippocampus (e.g., working memory; Finke et al., 2013) after surgical removal of active disease processes. However, others have shown that following temporal lobectomy, increased neural activity in the remnant hippocampal tail correlates negatively with episodic memory performance (Bonelli et al., 2013). This finding suggests that postoperative shrinkage of the remnant hippocampus (Baxendale et al., 2000) could offer an alternative basis for postoperative increases in neural activity during task performance than compensatory reorganization, at least in the operated hemisphere.

Together, our data provide novel evidence supporting the notion that altered MTL neural activity in disease is not intrinsically functionally efficient (Powell et al., 2007). It has rarely been considered whether neural activation patterns may differ according to whether patients are functionally impaired or relatively intact (as opposed to correlating neural activity with performance across the group). By directly comparing neural signals within dissociated hippocampal networks according to performance status, we demonstrate that chronic processes alone are insufficient to drive compensatory memory adaptation among functionally distinct MTL systems in the presence of active disease. Our findings highlight the importance of distinguishing the neural signatures of active disease-related processes from behaviorally efficient processes subserving reallocation of function.

\section{References}

Addis DR, Moscovitch M, McAndrews MP (2007) Consequences of hippocampal damage across the autobiographical memory network in left temporal lobe epilepsy. Brain 130:2327-2342. CrossRef Medline

Aggleton JP (2012) Multiple anatomical systems embedded within the primate medial temporal lobe: implications for hippocampal function. Neurosci Biobehav Rev 36:1579-1596. CrossRef Medline

Alessio A, Pereira FR, Sercheli MS, Rondina JM, Ozelo HB, Bilevicius E, Pedro T, Covolan RJ, Damasceno BP, Cendes F (2013) Brain plasticity for verbal and visual memories in patients with mesial temporal lobe epilepsy and hippocampal sclerosis: an fMRI study. Hum Brain Mapp 34:186199. CrossRef Medline

Bakker A, Krauss GL, Albert MS, Speck CL, Jones LR, Stark CE, Yassa MA, Bassett SS, Shelton AL, Gallagher M (2012) Reduction of hippocampal hyperactivity improves cognition in amnestic mild cognitive impairment. Neuron 74:467-474. CrossRef Medline

Banks SJ, Sziklas V, Sodums DJ, Jones-Gotman M (2012) fMRI of verbal and nonverbal memory processes in healthy and epileptogenic medial temporal lobes. Epilepsy Behav 25:42-49. CrossRef Medline

Baxendale SA, Thompson PJ, Kitchen ND (2000) Postoperative hippocampal remnant shrinkage and memory decline: a dynamic process. Neurology 55:243-249. CrossRef Medline

Bernhardt BC, Worsley KJ, Kim H, Evans AC, Bernasconi A, Bernasconi N (2009) Longitudinal and cross-sectional analysis of atrophy in pharmacoresistant temporal lobe epilepsy. Neurology 72:1747-1754. CrossRef Medline

Bettus G, Bartolomei F, Confort-Gouny S, Guedj E, Chauvel P, Cozzone PJ, Ranjeva JP, Guye M (2010) Role of resting state functional connectivity MRI in presurgical investigation of mesial temporal lobe epilepsy. J Neurol Neurosurg Psychiatry 81:1147-1154. CrossRef Medline

Bettus G, Ranjeva JP, Wendling F, Bénar CG, Confort-Gouny S, Régis J, Chauvel P, Cozzone PJ, Lemieux L, Bartolomei F, Guye M (2011) Interictal functional connectivity of human epileptic networks assessed by intracerebral EEG and BOLD signal fluctuations. PLoS One 6:e20071. CrossRef Medline

Blatt GJ, Rosene DL (1998) Organization of direct hippocampal efferent projections to the cerebral cortex of the rhesus monkey: projections from CA1, prosubiculum, and subiculum to the temporal lobe. J Comp Neurol 392:92-114. CrossRef Medline

Bonelli SB, Powell RH, Yogarajah M, Samson RS, Symms MR, Thompson PJ,
Koepp MJ, Duncan JS (2010) Imaging memory in temporal lobe epilepsy: predicting the effects of temporal lobe resection. Brain 133:11861199. CrossRef Medline

Bonelli SB, Thompson PJ, Yogarajah M, Powell RH, Samson RS, McEvoy AW, Symms MR, Koepp MJ, Duncan JS (2013) Memory reorganization following anterior temporal lobe resection: a longitudinal functional MRI study. Brain 136:1889-1900. CrossRef Medline

Bonnici HM, Sidhu M, Chadwick MJ, Duncan JS, Maguire EA (2013) Assessing hippocampal functional reserve in temporal lobe epilepsy: a multi-voxel pattern analysis of fMRI data. Epilepsy Res 105:140-149. CrossRef Medline

Brooks JC, Beckmann CF, Miller KL, Wise RG, Porro CA, Tracey I, Jenkinson M (2008) Physiological noise modelling for spinal functional magnetic resonance imaging studies. Neuroimage 39:680-692. CrossRef Medline

Clusmann H, Schramm J, Kral T, Helmstaedter C, Ostertun B, Fimmers R, Haun D, Elger CE (2002) Prognostic factors and outcome after different types of resection for temporal lobe epilepsy. J Neurosurg 97:1131-1141. CrossRef Medline

Concha L, Beaulieu C, Gross DW (2005) Bilateral limbic diffusion abnormalities in unilateral temporal lobe epilepsy. Ann Neurol 57:188-196. CrossRef Medline

Das SR, Mechanic-Hamilton D, Korczykowski M, Pluta J, Glynn S, Avants BB, Detre JA, Yushkevich PA (2009) Structure specific analysis of the hippocampus in temporal lobe epilepsy. Hippocampus 19:517-525. CrossRef Medline

Das SR, Mechanic-Hamilton D, Pluta J, Korczykowski M, Detre JA, Yushkevich PA (2011) Heterogeneity of functional activation during memory encoding across hippocampal subfields in temporal lobe epilepsy. Neuroimage 58:1121-1130. CrossRef Medline

Ewers M, Sperling RA, Klunk WE, Weiner MW, Hampel H (2011) Neuroimaging markers for the prediction and early diagnosis of Alzheimer's disease dementia. Trends Neurosci 34:430-442. CrossRef Medline

Fanselow MS, Dong HW (2010) Are the dorsal and ventral hippocampus functionally distinct structures? Neuron 65:7-19. CrossRef Medline

Figueiredo P, Santana I, Teixeira J, Cunha C, Machado E, Sales F, Almeida E, Castelo-Branco M (2008) Adaptive visual memory reorganization in right medial temporal lobe epilepsy. Epilepsia 49:1395-1408. CrossRef Medline

Finke C, Bruehl H, Düzel E, Heekeren HR, Ploner CJ (2013) Neural correlates of short-term memory reorganization in humans with hippocampal damage. J Neurosci 33:11061-11069. CrossRef Medline

Focke NK, Yogarajah M, Bonelli SB, Bartlett PA, Symms MR, Duncan JS (2008) Voxel-based diffusion tensor imaging in patients with mesial temporal lobe epilepsy and hippocampal sclerosis. Neuroimage 40:728 737. CrossRef Medline

Gour N, Ranjeva JP, Ceccaldi M, Confort-Gouny S, Barbeau E, Soulier E, Guye M, Didic M, Felician O (2011) Basal functional connectivity within the anterior temporal network is associated with performance on declarative memory tasks. Neuroimage 58:687-697. CrossRef Medline

Greve DN, Fischl B (2009) Accurate and robust brain image alignment using boundary-based registration. Neuroimage 48:63-72. CrossRef Medline

Gustin SM, Peck CC, Cheney LB, Macey PM, Murray GM, Henderson LA (2012) Pain and plasticity: is chronic pain always associated with somatosensory cortex activity and reorganization? J Neurosci 32:14874-14884. CrossRef Medline

He BJ, Snyder AZ, Zempel JM, Smyth MD, Raichle ME (2008) Electrophysiological correlates of the brain's intrinsic large-scale functional architecture. Proc Natl Acad Sci U S A 105:16039-16044. CrossRef Medline

Helmstaedter C (2004) Neuropsychological aspects of epilepsy surgery. Epilepsy Behav 5:S45-55. CrossRef Medline

Hirshhorn M, Grady C, Rosenbaum RS, Winocur G, Moscovitch M (2012) The hippocampus is involved in mental navigation for a recently learned, but not a highly familiar environment: a longitudinal fMRI study. Hippocampus 22:842-852. CrossRef Medline

Holloway V, Gadian DG, Vargha-Khadem F, Porter DA, Boyd SG, Connelly A (2000) The reorganization of sensorimotor function in children after hemispherectomy: a functional MRI and somatosensory evoked potential study. Brain 123:2432-2444. CrossRef Medline

Huijbers W, Pennartz CM, Cabeza R, Daselaar SM (2011) The hippocampus is coupled with the default network during memory retrieval but not during memory encoding. PLoS One 6:e17463. CrossRef Medline 
Johns P, Thom M (2008) Epilepsy and hippocampal sclerosis: cause or effect? ACNR 8:17-18.

Jokeit H, Okujava M, Woermann FG (2001) Carbamazepine reduces memory induced activation of mesial temporal lobe structures: a pharmacological fMRI-study. BMC Neurol 1:6. CrossRef Medline

Kahane P, Bartolomei F (2010) Temporal lobe epilepsy and hippocampal sclerosis: lessons from depth EEG recordings. Epilepsia 51:59-62. CrossRef Medline

Kahn I, Andrews-Hanna JR, Vincent JL, Snyder AZ, Buckner RL (2008) Distinct cortical anatomy linked to subregions of the medial temporal lobe revealed by intrinsic functional connectivity. J Neurophysiol 100: 129-139. CrossRef Medline

Laufs H, Hamandi K, Salek-Haddadi A, Kleinschmidt AK, Duncan JS, Lemieux L (2007) Temporal lobe interictal epileptic discharges affect cerebral activity in "default mode" brain regions. Hum Brain Mapp 28:1023-1032. CrossRef Medline

Libby LA, Ekstrom AD, Ragland JD, Ranganath C (2012) Differential connectivity of perirhinal and parahippocampal cortices within human hippocampal subregions revealed by high-resolution functional imaging. J Neurosci 32:6550-6560. CrossRef Medline

Makin TR, Scholz J, Filippini N, Henderson Slater D, Tracey I, Johansen-Berg H (2013) Phantom pain is associated with preserved structure and function in the former hand area. Nat Commun 4:1570. CrossRef Medline

McCormick C, Quraan M, Cohn M, Valiante TA, McAndrews MP (2013) Default mode network connectivity indicates episodic memory capacity in mesial temporal lobe epilepsy. Epilepsia 54:809-818. CrossRef Medline

Moser MB, Moser EI (1998) Functional differentiation in the hippocampus. Hippocampus 8:608-619. CrossRef Medline

O’Reilly JX, Beckmann CF, Tomassini V, Ramnani N, Johansen-Berg H (2010) Distinct and overlapping functional zones in the cerebellum defined by resting state functional connectivity. Cereb Cortex 20:953-965. CrossRef Medline

Petrides M, Pandya DN (1999) Dorsolateral prefrontal cortex: comparative cytoarchitectonic analysis in the human and the macaque brain and corticocortical connection patterns. Eur J Neurosci 11:1011-1036. CrossRef Medline

Poppenk J, Moscovitch M (2011) A hippocampal marker of recollection memory ability among healthy young adults: contributions of posterior and anterior segments. Neuron 72:931-937. CrossRef Medline

Poppenk J, McIntosh AR, Craik FI, Moscovitch M (2010) Past experience modulates the neural mechanisms of episodic memory formation. J Neurosci 30:4707-4716. CrossRef Medline

Powell HW, Richardson MP, Symms MR, Boulby PA, Thompson PJ, Duncan JS, Koepp MJ (2007) Reorganization of verbal and nonverbal memory in temporal lobe epilepsy due to unilateral hippocampal sclerosis. Epilepsia 48:1512-1525. CrossRef Medline

Powell HW, Richardson MP, Symms MR, Boulby PA, Thompson PJ, Duncan JS, Koepp MJ (2008) Preoperative fMRI predicts memory decline following anterior temporal lobe resection. J Neurol Neurosurg Psychiatry 79:686-693. CrossRef Medline

Proper EA, Oestreicher AB, Jansen GH, Veelen CW, van Rijen PC, Gispen WH, de Graan PN (2000) Immunohistochemical characterization of mossy fibre sprouting in the hippocampus of patients with pharmacoresistant temporal lobe epilepsy. Brain 123:19-30. CrossRef Medline

Putcha D, Brickhouse M, O'Keefe K, Sullivan C, Rentz D, Marshall G, Dickerson B, Sperling R (2011) Hippocampal hyperactivation associated with cortical thinning in Alzheimer's disease signature regions in nondemented elderly adults. J Neurosci 31:17680-17688. CrossRef Medline Rabin ML, Narayan VM, Kimberg DY, Casasanto DJ, Glosser G, Tracy JI,
French JA, Sperling MR, Detre JA (2004) Functional MRI predicts postsurgical memory following temporal lobectomy. Brain 127:2286-2298. CrossRef Medline

Saur D, Hartwigsen G (2012) Neurobiology of language recovery after stroke: lessons from neuroimaging studies. Arch Phys Med Rehab 93:S1525. CrossRef Medline

Schwarze U, Hahn C, Bengner T, Stodieck S, Büchel C, Sommer T (2009) Enhanced activity during associative encoding in the affected hippocampus in right temporal lobe epilepsy patients. Brain Res 1297:112-117. CrossRef Medline

Sestieri C, Corbetta M, Romani GL, Shulman GL (2011) Episodic memory retrieval, parietal cortex, and the default mode network: functional and topographic analyses. J Neurosci 31:4407-4420. CrossRef Medline

Sperling MR, O'Connor MJ (1990) Auras and subclinical seizures: characteristics and prognostic significance. Ann Neurol 28:320-328. CrossRef Medline

Stagg CJ, Bachtiar V, O'Shea J, Allman C, Bosnell RA, Kischka U, Matthews PM, Johansen-Berg H (2012) Cortical activation changes underlying stimulation-induced behavioural gains in chronic stroke. Brain 135:276284. CrossRef Medline

Sutula T, Cascino G, Cavazos J, Parada I, Ramirez L (1989) Mossy fiber synaptic reorganization in the epileptic human temporal lobe. Ann Neurol 26:321-330. CrossRef Medline

Thom M, Eriksson S, Martinian L, Caboclo LO, McEvoy AW, Duncan JS, Sisodiya SM (2009) Temporal lobe sclerosis associated with hippocampal sclerosis in temporal lobe epilepsy: neuropathological features. J Neuropathol Exp Neurol 68:928-938. CrossRef Medline

Vann SD, Aggleton JP, Maguire EA (2009) What does the retrosplenial cortex do? Nat Rev Neurosci 10:792-802. CrossRef Medline

Vargha-Khadem F, Isaacs EB, Papaleloudi H, Polkey CE, Wilson J (1991) Development of language in six hemispherectomized patients. Brain 114: 473-495. CrossRef Medline

Vincent JL, Snyder AZ, Fox MD, Shannon BJ, Andrews JR, Raichle ME, Buckner RL (2006) Coherent spontaneous activity identifies a hippocampal-parietal memory network. J Neurophysiol 96:3517-3531. CrossRef Medline

Voets NL, Adcock JE, Stacey R, Hart Y, Carpenter K, Matthews PM, Beckmann CF (2009) Functional and structural changes in the memory network associated with left temporal lobe epilepsy. Hum Brain Mapp 30: 4070-4081. CrossRef Medline

Voets NL, Bernhardt BC, Kim H, Yoon U, Bernasconi N (2011) Increased temporolimbic cortical folding complexity in temporal lobe epilepsy. Neurology 76:138-144. CrossRef Medline

Wang L, Laviolette P, O'Keefe K, Putcha D, Bakkour A, Van Dijk KR, Pihlajamäki M, Dickerson BC, Sperling RA (2010) Intrinsic connectivity between the hippocampus and posteromedial cortex predicts memory performance in cognitively intact older individuals. Neuroimage 51:910 917. CrossRef Medline

Zamboni G, Wilcock GK, Douaud G, Drazich E, McCulloch E, Filippini N, Tracey I, Brooks JC, Smith SM, Jenkinson M, Mackay CE (2013) Resting functional connectivity reveals residual functional activity in Alzheimer's disease. Biol Psychiatry 74:375-383. CrossRef Medline

Zarei M, Beckmann CF, Binnewijzend MA, Schoonheim MM, Oghabian MA, Sanz-Arigita EJ, Scheltens P, Matthews PM, Barkhof F (2012) Functional segmentation of the hippocampus in the healthy human brain and in Alzheimer's disease. Neuroimage 66:28-35. CrossRef Medline

Zatorre RJ, Fields RD, Johansen-Berg H (2012) Plasticity in gray and white: neuroimaging changes in brain structure during learning. Nat Neurosci 15:528-536. CrossRef Medline 